\title{
MicroSEC: Sequence error filtering pipeline for formalin-fixed and paraffin-embedded samples
}

Masachika Ikegami ( $\nabla$ ikegami-tky@umin.ac.jp )

National Cancer Center Research Institute

\section{Shinji Kohsaka}

Division of Cellular Signaling, National Cancer Center Research Institute

\section{Takeshi Hirose}

Division of Cellular Signaling, National Cancer Center Research Institute

\section{Toshihide Ueno}

Division of Cellular Signaling, National Cancer Center Research Institute

\section{Naoki Kanomata}

Department of Pathology, St Luke's International Hospital

\section{Hideko Yamauchi}

Department of Breast Surgical Oncology, St Luke's International Hospital

Hiroshi Kobayashi

Department of Orthopaedic Surgery, Faculty of Medicine, The University of Tokyo

\section{Sakae Tanaka}

The University of Tokyo

Hiroyuki Mano

National Cancer Center Research Institute https://orcid.org/0000-0003-4645-0181

\section{Brief Communication}

Keywords: formalin-fixed, paraffin-embedded samples, MicroSEC, sequencing errors

Posted Date: January 28th, 2021

DOI: https://doi.org/10.21203/rs.3.rs-153650/v1

License: (c) (i) This work is licensed under a Creative Commons Attribution 4.0 International License. Read Full License

Version of Record: A version of this preprint was published at Communications Biology on December 15th, 2021. See the published version at https://doi.org/10.1038/s42003-021-02930-4. 


\section{Brief Communication}

2 MicroSEC: Sequence error filtering pipeline for formalin-fixed and paraffin-embedded 3 samples

5 Authors: Masachika Ikegami ${ }^{1,2,3, *}$, Shinji Kohsaka ${ }^{1, *}$, Takeshi Hirose ${ }^{1,4}$, Toshihide Ueno ${ }^{1}$,

6 Naoki Kanomata ${ }^{5}$, Hideko Yamauchi ${ }^{6}$, Hiroshi Kobayashi ${ }^{2}$, Sakae Tanaka ${ }^{2}$, and Hiroyuki

7 Mano $^{1, *}$

8 Affiliations:

$9 \quad{ }^{1}$ Division of Cellular Signaling, National Cancer Center Research Institute, Tokyo, Japan

$10{ }^{2}$ Department of Orthopaedic Surgery, Faculty of Medicine, The University of Tokyo, Tokyo, 11 Japan

$12{ }^{3}$ Department of Musculoskeletal Oncology, Tokyo Metropolitan Cancer and Infectious

13 Diseases Center Komagome Hospital, Tokyo, Japan

$14{ }^{4}$ Department of Orthopaedic Surgery, Graduate School of Medical Sciences, Kyushu

15 University, Fukuoka, Japan

16 5epartment of Pathology, St Luke's International Hospital, Tokyo, Japan

$17{ }^{6}$ Department of Breast Surgical Oncology, St Luke's International Hospital, Tokyo, Japan

*Correspondence should be addressed to: Masachika Ikegami (ikegami-tky@umin.ac.jp),

20 Shinji Kohsaka (skohsaka@ncc.go.jp), or Hiroyuki Mano (hmano@ncc.go.jp).

Corresponding authors:

Masachika Ikegami

24 Division of Cellular Signaling, National Cancer Center Research Institute, 5-1-1 Tsukiji,

25 Chuo-ku, Tokyo 104-0045, Japan 
26 Tel.: +81-3- 3547-5201; Fax: +81-3- 5565-0727; Email: ikegami-tky@umin.ac.jp 27

28 Shinji Kohsaka

29 Division of Cellular Signaling, National Cancer Center Research Institute, 5-1-1 Tsukiji,

30 Chuo-ku, Tokyo 104-0045, Japan

31 Tel.: +81-3- 3547-5201; Fax:+81-3- 5565-0727; Email: skohsaka@ncc.go.jp

32

33 Hiroyuki Mano

34 Division of Cellular Signaling, National Cancer Center Research Institute, 5-1-1 Tsukiji,

35 Chuo-ku, Tokyo 104-0045, Japan

36 Tel.: +81-3- 3547-5241; Fax: +81-3-3248-0326; Email: hmano@ncc.go.jp 


\section{Abstract}

38 The clinical sequencing of tumors is usually performed on formalin-fixed, paraffin-embedded

39 (FFPE) samples and results in many sequencing errors. Most of these errors are detected in

40 chimeric reads caused by single-strand DNA molecules with microhomology. Our filtering

41 pipeline, MicroSEC, focuses on the uneven distribution of mutations in each read and

42 removes the sequencing errors in FFPE samples without eliminating the true mutations that

43 are also detected in fresh frozen samples.

44

45 
46 Cancer gene panel testing using next-generation sequencing has been applied in routine

47 practice to identify the somatic as well as germline mutations and to determine the

48 appropriate treatment strategy for cancer patients ${ }^{1}$. Somatic mutations often only occur in a

49 small subset of cells and are present in a small fraction of DNA molecules from tumor

50 samples. Somatic mutations can be detected in fresh or fresh frozen (FF) samples with the

51 next-generation sequencers, but those materials are not always available for clinical

52 sequencing.

As a result, the nucleic acids extracted from formalin-fixed and paraffin-embedded

54 (FFPE) tumor tissues collected in surgeries or biopsies are more commonly used ${ }^{2-4}$. Formalin

55 fixation and prolonged storage cause various changes on nucleic acids, such as the cross-

56 linking between nucleic acids and proteins, denaturation, cytosine deamination, and chemical

57 modification ${ }^{5}$. As a result, the DNA extracted from FFPE tissues is usually fragmented and

58 contains single-stranded DNA (ssDNA) ${ }^{6}$. Low DNA quality causes significant noise in the

59 sequencing reaction. Therefore, it is highly challenging to detect the mutations that occur at

60 low variant allele fractions (VAF) in FFPE samples ${ }^{7-9}$. However, the development of filtering

61 pipelines for FFPE artifacts has not progressed significantly beyond the well-known CG-to-

62 TG mutation caused by cytosine deamination ${ }^{10-14}$.

63 Here, we propose a major mechanism for FFPE sequencing errors, microhomology-

64 induced chimeric read (MICR) formation in the capture-based target sequencing. MICRs are 
ssDNA-derived artifacts and classified into two types induced by different mechanisms. The

66 first type of MICRs result from the hairpin structure formed by two palindromic sequences

67 in the same ssDNA molecule. The second type of MICRs is formed from the mis-annealing

68 of two ssDNA molecules derived from different homologous regions (Fig. 1a-c and

69 Supplementary Fig. 1).

MICRs are formed during the end-repair step in the library preparation for clinical

71 sequencing, when a considerable amount of extracted DNA is denatured to ssDNA and

72 behaves as site-directed mutagenesis polymerase chain reaction (PCR) primers ${ }^{15}$. In the case

73 of a true mutation, the altered bases are expected to be evenly distributed across the reads.

74 Conversely, in case of an MICR error, the altered bases are distributed preferentially near the

75 effective ends of the read, with severely limited lengths that match the reference sequence

76 upstream or downstream of the mutations. VarScan2 mutation caller employs it as a filtering

77 metric $^{16}$. However, such bias is vaguely known. As MICRs form before the adapter ligation

78 step in the library preparation, it cannot be ruled out in principle using template tagmentation

79 techniques such as molecular identifier (ID) barcoding ${ }^{1}$. Therefore, there is a significnt need

80 for specifically designed filtering pipelines.

81 Based on our theory that artifacts are derived from ssDNA-annealing, we have

82 developed a MICR-originating Sequence Error Cleaning pipeline (MicroSEC), a post hoc

83 filtering pipeline to predict if a given mutation is an MICR-derived error, utilizing BAM files 
84 and lists of mutation-supporting read IDs. MicroSEC is based on three criteria (Fig. 1d and

85 Online Methods). First, the local palindromic sequences causing hairpin-induced errors are

86 detected (Filter 2). Second, the distribution of mutation positions in sequencing reads is

87 interrogated if it is too uneven to occur probabilistically (Filter 1 and 3). Third, distant

88 regions in the genome are searched, of which sequences are exactly matched to the query

89 FFPE reads (Filter 4).

90 During the step of detecting hairpin-induced errors, 15-base sequences containing the

91 mutation are extracted from each read. We consider the mutation an artifact if more than half

92 of the sequences existed on the opposite strand within 150 bases of the neighboring sequence.

93 During the step of detecting mutated base position bias, the number of bases from the

94 mutated base to the farthest mapped base in each read is defined as the 3'or 5'-supporting

95 lengths. The probability of the mutation distribution of the supporting lengths is calculated

96 based on the multinomial distribution; mutations with $p<10^{-6}$ are considered artifacts.

97 During the step of detecting distant homologous regions, 40-base sequences including the

98 mutation are extracted from each read. The mutation is considered an artifact if more than

$99 \quad 15 \%$ of the sequences match another region in the genome.

100 Then, we examined the sensitivity of MicroSEC in distinguishing true mutations from

101 FFPE artifacts. We analyzed the target sequencing data of 53 FF and 190 FFPE normal breast

102 tissue samples with a high mean coverage of 400 or more. Our initial somatic mutation 
analysis pipeline identified an average of 0.3 and 11.8 mutations per sample in FF and FFPE

mutations per sample were filtered out from the data of the FF and FFPE samples,

mutations in or adjacent to a homopolymer equal to or longer than 10-base, accounted for through the filter had similar VAFs to the filtered-out mutations (Supplementary Fig. 2a).

110 Surprisingly, two unique mutations with VAF of $>50 \%$ in FFPE samples were

111 eliminated by Filter 1 or 2 (Supplementary Table 1). Thus, a high VAF does not necessarily

112 indicate the mutation as a true one. The adequate threshold of mutation depth was also

113 examined using FFPE samples from normal breast tissues, which were expected to have few

114 or no mutations. When there were 10 or more reads supporting mutations, more than $90 \%$ of

115 the mutations were consistently filtered out (Supplementary Fig. 3).

116 We then tested the specificity of MicroSEC by analyzing the target sequencing data of

11723 FF and 33 FFPE breast cancer samples with a high mean coverage, including eight sets of

118 matched FF and FFPE samples from the same patients. Our pipeline identified 4.0 and 7.5

119 mutations per sample in the FF and FFPE samples, respectively (Table 1). All the mutations

120 in the FF samples passed through the filter, while 3.2 mutations per sample $(30.1 \%)$ were

121 filtered out in the FFPE samples. Mutations passing through the filter tended to have a higher 
122 VAF than the filtered mutations (Supplementary Fig. 2b). Notably, MicroSEC did not

123 remove any mutations detected in matched FF samples (Supplementary Fig. 4). We revealed

124 the different distribution of the rates among low-quality bases, soft-clipped reads, artifacts

125 from homologous regions, and mutations derived from hairpin structures in the FF and FFPE

126 samples. We also confirmed that the thresholds used for filtering were appropriate

127 (Supplementary Fig. 5).

128 The MicroSEC analysis results were validated with amplicon-based sequencing that

129 enriches target genomic regions by PCR. Thirty mutations including germline mutations and

130 low VAF ones, found in the breast tissue samples, were examined. In theory, MICR-

131 originated artifacts cannot occur in amplification-based sequencing because PCR does not

132 amplify small structures formed by microhomology. Consistent with this prediction, 11

133 mutations that passed through the MicroSEC filter were all detected by amplicon-based

134 sequencing with a similar level of VAF, whereas 14 filtered mutations were not detected by

135 amplicon-based sequencing (Fig. 1e and Supplementary Table 2). A CG-to-TG mutation with

$1365.5 \%$ of VAF was not amplified in amplicon-based sequencing because this artifact arose

137 from deaminated cytosine, and the Q5 DNA polymerase could not amplify such degenerated 138 template DNA.

139 We further examined the utility of MicroSEC in the clinical setting by re-analyzing

140 the sequencing data of 54 clinical FFPE samples with a high mean coverage (Table 1$)^{2}$. At 
141 first, 21.7 somatic mutations per sample were detected by the conventional mutation caller

142 pipeline. MicroSEC detected 3.7 artifacts per sample (16.9\%), including five unique

143 pathogenic mutations (Supplementary Table 3). Since annotating artifacts as pathogenic can

144 compromise the reliability of the clinical sequencing, the post hoc filtering with MicroSEC is

145 of great value.

146 Most other existing error detection algorithms utilize strand bias, k-mer frequency,

147 suffix trie, multiple sequence alignment, and statistical error models ${ }^{12,13}$. The application of

148 these algorithms is limited because they are not highly accurate. These methods disregard

149 detailed genomic information such as the local palindromic sequences and the position of

150 mutations within reads. A few pipelines are constructed to remove the chimeric reads

151 generated by PCR, only during the sequencing of about 1.5 kilobase pairs of the $16 \mathrm{~S}$

152 ribosomal $\mathrm{RNA}^{14,17,18}$.

153 In contrast, MicroSEC utilizes such overlooked information. MicroSEC's focus on the

154 distribution of mutations in each read enables it to remove only FFPE artifacts without

155 eliminating the true mutations. The effectiveness of MicroSEC is similar to the experimental

156 reduction of FFPE artifacts by degrading ssDNA with S1 nuclease ${ }^{6}$. MicroSEC is useful in

157 applications in target deep sequencing or whole-exome sequencing data with very high

158 coverage. A caveat of MicroSEC lies in its basis on probability calculations; therefore, it

159 requires a high number of reads supporting each mutation. Overall, our pipeline will increase 
160 the reliability of the studies that use FFPE samples, thus advancing cancer research

161 significantly. Our algorithm can also help to convey the correct information to cancer patients

162 who underwent clinical sequencing, resulting in the improvement of quality and precision of

163 their treatments. The source code of MicroSEC is freely available at

164 https://github.com/MANO-B/MicroSEC.

\section{ONLINE METHODS}

\section{Specimens}

167 The study cohort comprised 26 patients with breast cancer who underwent tumor resection or

168 prophylactic surgery because of hereditary breast and ovarian cancer syndrome at St. Luke's

169 International Hospital between 2010 and 2020. A pathologist (N.K.) specializing in breast

170 cancer reviewed the histological features and location of the tumors. Fifty-six FF samples of

171 normal breast tissue were obtained from 14 patients, and 211 FFPE samples were obtained

172 from 26 patients. Twenty-three FF breast cancer samples were obtained from nine patients,

173 and 37 FFPE samples were obtained from 11 patients, including matching FF and FFPE

174 samples from eight patients. The FFPE specimens were fixed in 10\% neutral-buffered

175 formalin for 15-72 hours and then embedded in paraffin blocks. All FFPE specimens had

176 been stored at room temperature for at least six months before DNA extraction. Blood

177 samples were obtained from all the patients as a source of matching normal DNA. 
179 Hospital and the National Cancer Center Research Institute. Written informed consent was

180 obtained from all the participants.

\section{Capture-based panel sequencing}

182 Genomic DNA was isolated from FF or blood samples using QIAamp DNA Mini Kits

183 (Qiagen, Germany) or FFPE samples using GeneRead DNA FFPE Kits (Qiagen, Germany).

184 Then, uracil DNA glycosylase treatment was performed for FFPE samples to remove C-to-T

185 artifacts. Lastly, 500 ng of genomic DNA was subjected to target fragment enrichment using

186 SureSelectXT Custom kits (Agilent Technologies, USA) or Twist Custom Panels (Twist

187 Bioscience, USA). Custom-made probes were designed to hybridize and capture the target

188 genes listed in the Todai OncoPanel (TOP) as previously described ${ }^{2}$. Double-stranded linear

189 DNA libraries for Illumina sequencer were converted to single-stranded circular DNA

190 libraries for MGI sequencer with the MGIEasy Universal Library Conversion Kit (MGI

191 Technologies, China). A library's quantity and quality were assessed with a Qubit 2.0

192 Fluorometer (Thermo Fisher Scientific, USA) and the Agilent 2200 TapeStation System

193 (Agilent Technologies, USA). The massive parallel sequencing of the isolated fragments with

194 124-151 base pair read lengths was performed using the HiSeq 2500 System, NovaSeq 6000

195 System (Illumina, USA), or DNBSEQ-G400 sequencer (MGI Technologies, China). Samples

196 with an insufficient mean coverage of less than 400 were excluded $(n=28,8.6 \%)$. 


\section{Amplicon-based sequencing}

198 The genomic regions of 106-156 bases around a mutation were amplified by PCR using the

199 NEB Q5 Hot Start HiFi PCR Master Mix (New England BioLabs, USA) and appropriate

200 primer sets (Supplementary Table 3). PCR products were purified using Agencourt AMPure

201 XP beads (Beckman Coulter, USA) and subjected to library preparation with the NEBNext

202 Ultra II DNA Library Prep Kit for Illumina (New England BioLabs, USA). The libraries were

203 sequenced to generate 150 base paired-end reads using the Miseq system (Illumina, USA).

\section{Somatic mutation calls}

205 Paired-end reads were independently aligned to the human reference genome (University of

206 California, Santa Cruz Genome Browser assembly ID: hg38) using Burrows-Wheeler Aligner

$207(\mathrm{v} 0.7 .17)^{19}$ and Bowtie2 (v2.1.0) ${ }^{20}$. Potential PCR duplicates were removed with SAMtools

$208(\mathrm{v} 1.9)^{21}$. Somatic mutations were identified using MuTect2 (GATK v4.1.3.0) ${ }^{22}$, VarScan2

$209(\mathrm{v} 2.4 .3)^{16}$, and our in-house pipeline.

210 Mutations were discarded if the read depth was shorter than 100 base pairs or the

211 number of mutation-supporting reads was fewer than 10 or VAF was less than 5\%. Mutations

212 were also eliminated if the reads were supported by only one strand of the chromosome,

213 observed in normal human genomes in the 1000 Genomes Project dataset ${ }^{23}$ with a frequency

214 greater than $1 \%$ or in our in-house database, located outside the 3.4 megabase TOP target

215 region, or located at simple repeat sequences downloaded from the Tandem Repeats Database 
216 (https://tandem.bu.edu/cgi-bin/trdb/trdb.exe) $)^{24,25}$. In addition, the mutations were removed if

217 a homopolymer consisting of more than 14 bases was present within 20 bases around the

218 mutation; or more than $10 \%$ of the bases within the mutation supporting reads had a Phred

219 quality score of less than Q18. The mapped reads and called mutations were visualized and

220 manually checked with the Integrative Genomics Viewer (v2.4.10) ${ }^{26}$.

\section{MicroSEC pipeline}

222 MicroSEC (v1.1.18) is a filtering pipeline written in R language designed to discover MICR-

223 derived sequencing errors in FFPE samples. It detects local palindromic sequences, the

224 uneven distribution of mutated bases within each read, and the pseudo-mutations introduced

225 by distant homologous regions.

226 The workflow of MicroSEC in this study was composed of several steps. First, the

227 positions of the mutated bases were detected with the matchPattern function from the

228 Biostrings package (v2.54.0). Second, the adapter sequence at the 3' end of each read was

229 trimmed with the trimLRPatterns function from the Biostrings package with the maximum

230 mismatch rate of $10 \%$. Then, the distance from the altered base to the read's effective ends

231 was calculated based on the Compact Idiosyncratic Gapped Alignment Report (CIGAR)

232 strings of the alignment results (see "Supporting length analysis"). Next, the hairpin structure-

233 induced chimeric reads were detected (see "Hairpin structure detection"). Afterward, the

234 entire genome was searched for regions that were homologous to the sequence around the 
235 mutation using the countPDict function from the Biostrings package (see "Homologous

236 region detection"). Lastly, the above results were combined to construct the MicroSEC

237 filtering pipeline. The results of MicroSEC's filtering of the somatic mutations in breast

238 tissue samples and clinical sequencing samples were provided as Supplementary Data.

\section{Supporting length analysis}

240 It is essential to identify the uneven distribution of the mutations in the mutation-supporting

241 reads for discriminating between the MICR-derived errors and true mutations. Here, we have

242 introduced the concept of support lengths, which are the base lengths from the mutated bases

243 (excluding the mutated bases) to the furthest mapped bases in each read, based on the CIGAR

244 strings in BAM files. When a mutation is a substitution of a number $(\mathrm{N})$ of nucleotides, and

245 the read length is another number (L) of nucleotides, the 3 '- or 5'-supporting lengths are

246 distributed between zero and $\mathrm{L}-\mathrm{N}$. When a mutation is an N-nucleotide deletion or

247 insertion, the possibility of the mutation being the part of a repeated sequence should be

248 considered, because the mutation-supporting reads must contain all the repetitive sequence to

249 determine the presence or absence of small insertions/deletions (indels).

250 Furthermore, we need to consider the penalties by Burrows-Wheeler Aligner for

251 mapping with gaps. Since the penalty due to an N-base gap is $\mathrm{N}+6$ and the soft-clipping

252 penalty is 5 , a sequence supporting the indel would be soft-clipped if there are no more than

$253 \mathrm{~N}+1$ matching sequences outside the gap. Given the $5^{\prime}$ and 3 ' repetitive sequences around 
254 the mutation of lengths $\mathrm{R}_{5^{\prime}}$ and $\mathrm{R}_{3^{\prime}}$, the supporting lengths can be distributed between $\max \left(\mathrm{R}_{3^{\prime}}\right.$

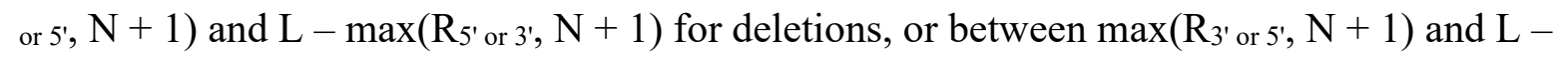

$256 \mathrm{~N}-\max \left(\mathrm{R}_{5^{\prime}}\right.$ or $\left.3^{\prime}, \mathrm{N}+1\right)$ for insertions, with $\max (\mathrm{X}, \mathrm{Y})$ denoting the larger value of $\mathrm{X}$ and $\mathrm{Y}$.

258 shorter of the 3'- and 5'-supporting lengths in each read. The theoretical distributions are and between $\min \left(\max \left(\mathrm{R}_{3^{\prime}}, \mathrm{N}+1\right), \max \left(\mathrm{R}_{5^{\prime}}, \mathrm{N}+1\right)\right)$ and $[(\mathrm{L}-\mathrm{N}) / 2]$, for substitutions, deletions, and insertions, respectively. $[\mathrm{X}]$ represents the greatest integer less than or equal to $\mathrm{X}$, with $\min (\mathrm{X}, \mathrm{Y})$ denoting the smaller value of $\mathrm{X}$ and $\mathrm{Y}$.

264 distribution is between $\mathrm{C}$ and $\mathrm{D}$, and the coverage depth of the mutation-supporting reads is

265 E. In that case, the probability $p$ is calculated based on the multinomial distribution by the

266 following equations:

$p=\left\{\sum_{m=A}^{B} f(m) / \sum_{n=C}^{D} f(n)\right\}^{E}$

$268 f(x)=[$ The number of reads of which the supporting length is $x$.]

269 We regard mutations with $p<10^{-6}$ as errors in principle. During deep sequencing, however,

$270 p$ becomes too small with large E, leading to the overfiltering of the mutations. Thus, the

271 filter is not applied to the mutations if $\mathrm{B}-\mathrm{A}+1$ is greater than $75 \%$ of $\mathrm{D}-\mathrm{C}+1$, or 
274 length will increase because of the cumulative mismatch penalties of the mutations.

275 Overfiltering can be avoided by fixing the minimum supporting length to zero if there are

276 more than three mismatched bases in the 10 bases surrounding the mutation.

\section{Hairpin structure detection}

278 A sequence of at least 15 consecutive bases, with the mutated bases at the center, was

279 extracted from each read. If the sequence was present on the opposite strand and within 150

280 bases of the neighboring sequence, the mutation was suspected to be derived from a hairpin

281 structure. If more than $50 \%$ of the reads had such sequences, the mutation would be

282 considered an error.

\section{Homologous region detection}

284 We extracted a sequence comprising the mutated bases and the surrounding repetitive

285 sequences if available from each read. Four upstream or downstream bases were added to that

286 sequence, and a sequence further from the sequence with the mutation was added on the

287 opposite side to create two sequences with a total of 40 bases. We searched the entire genome

288 for regions matching exactly to one of the two sequences. If more than $15 \%$ of the reads had

289 such homologous sequences, the mutation would be considered an artifact.

\section{Data availability}


291 We have deposited the raw sequencing data of the breast tissue samples examined in this

292 study under the accession number JGASxxxxxxxxxx (accession codes will be available

293 before publication) in the Japanese Genotype-Phenotype Archive

294 (http://trace.ddbj.nig.ac.jp/jga) hosted by the DNA Data Bank of Japan. The target

295 sequencing data of 54 cancer specimens are available for download at the Japanese

296 Genotype-Phenotype Archive under the accession number JGAS00000000164.

297 Code availability

298 All the source code written in R for MISEC is publicly available at

299 https://github.com/MANO-B/MicroSEC and https://cran.r-

300 project.org/web/packages/MicroSEC.

301

302 Acknowledgments

303 The authors would like to thank A. Shiino for technical assistance. The study was supported

304 in part by the World-leading Innovative Graduate Study Program for Life Science and

305 Technology, The University of Tokyo, as part of the WISE Program (Doctoral Program for

306 World-leading Innovative \& Smart Education), MEXT, Japan; JSPS KAKENHI grants

307 (\#19J13207); Grant of Japan Orthopaedics and Traumatology Research Foundation (No.

308 418); AMED under Grant Number JP20ae0101074.

309

310 Author contributions

311 M.I. conceived the project and developed the algorithm. N.K. and H.Y. collected breast tissue

312 samples. M.I., S.K., T.H., and T.U. designed and analyzed the experiments. H.K. and S.T. 
313 supervised the projects. M.I., S.K., T.H., T.U., and H.M. wrote the manuscript with input

314 from all authors. All authors have read and approved the manuscript.

315

\section{Competing interests}

317 The authors declare no competing interests.

318

319 References

320 1. Singh, R.R. Next-Generation Sequencing in High-Sensitive Detection of Mutations in Tumors: Challenges, Advances, and Applications. J Mol Diagn 22, 994-1007 (2020).

322

2. Kohsaka, S. et al. Comprehensive assay for the molecular profiling of cancer by target enrichment from formalin - fixed paraffin - embedded specimens. Cancer Science 110,

3. Zehir, A. et al. Mutational landscape of metastatic cancer revealed from prospective clinical sequencing of 10,000 patients. Nat Med 23, 703-713 (2017).

4. Cheng, D.T. et al. Memorial Sloan Kettering-Integrated Mutation Profiling of Actionable Cancer Targets (MSK-IMPACT): A Hybridization Capture-Based NextGeneration Sequencing Clinical Assay for Solid Tumor Molecular Oncology. $J$ Mol

331 5. Do, H. \& Dobrovic, A. Sequence artifacts in DNA from formalin-fixed tissues: causes and strategies for minimization. Clin Chem 61, 64-71 (2015).

333 6. Haile, S. et al. Sources of erroneous sequences and artifact chimeric reads in next 
generation sequencing of genomic DNA from formalin-fixed paraffin-embedded samples. Nucleic Acids Research 47, e12-e12 (2019).

336 7. de Schaetzen van Brienen, L. et al. Comparative analysis of somatic variant calling on matched FF and FFPE WGS samples. BMC Med Genomics 13, 94 (2020).

8. Astolfi, A. et al. Whole exome sequencing (WES) on formalin-fixed, paraffinembedded (FFPE) tumor tissue in gastrointestinal stromal tumors (GIST). BMC Genomics 16, 892 (2015).

341 9. Wang, M. et al. Somatic Mutation Screening Using Archival Formalin-Fixed, ParaffinEmbedded Tissues by Fluidigm Multiplex PCR and Illumina Sequencing. J Mol Diagn 17, 521-532 (2015).

10. Bhagwate, A.V. et al. Bioinformatics and DNA-extraction strategies to reliably detect genetic variants from FFPE breast tissue samples. BMC Genomics 20, 689 (2019).

346 11. Spencer, D.H. et al. Comparison of clinical targeted next-generation sequence data from formalin-fixed and fresh-frozen tissue specimens. J Mol Diagn 15, 623-633 (2013).

348 12. Laehnemann, D., Borkhardt, A. \& McHardy, A.C. Denoising DNA deep sequencing data-high-throughput sequencing errors and their correction. Briefings in Bioinformatics 17, 154-179 (2016).

351 13. Lee, B., Moon, T., Yoon, S. \& Weissman, T. DUDE-Seq: Fast, flexible, and robust denoising for targeted amplicon sequencing. PLoS One 12, e0181463 (2017). 
353 14. Quince, C., Lanzen, A., Davenport, R.J. \& Turnbaugh, P.J. Removing noise from pyrosequenced amplicons. BMC Bioinformatics 12, 38 (2011).

355 15. Antikainen, N.M. \& Martin, S.F. Altering protein specificity: techniques and applications. Bioorg Med Chem 13, 2701-2716 (2005).

16. Koboldt, D.C. et al. VarScan 2: somatic mutation and copy number alteration discovery in cancer by exome sequencing. Genome Res 22, 568-576 (2012).

17. Haas, B.J. et al. Chimeric 16S rRNA sequence formation and detection in Sanger and 454-pyrosequenced PCR amplicons. Genome Res 21, 494-504 (2011).

18. Sze, M.A. \& Schloss, P.D. The Impact of DNA Polymerase and Number of Rounds of Amplification in PCR on 16S rRNA Gene Sequence Data. mSphere 4 (2019).

19. Li, H. \& Durbin, R. Fast and accurate short read alignment with Burrows-Wheeler transform. Bioinformatics 25, 1754-1760 (2009).

20. Langmead, B. \& Salzberg, S.L. Fast gapped-read alignment with Bowtie 2. Nat Methods 9, 357-359 (2012).

21. Li, H. et al. The Sequence Alignment/Map format and SAMtools. Bioinformatics 25, 2078-2079 (2009).

22. McKenna, A. et al. The Genome Analysis Toolkit: a MapReduce framework for analyzing next-generation DNA sequencing data. Genome Res 20, 1297-1303 (2010). 

68-74 (2015).

373 24. Benson, G. Tandem repeats finder: a program to analyze DNA sequences. Nucleic Acids Res 27, 573-580 (1999).

375

25. Gelfand, Y., Rodriguez, A. \& Benson, G. TRDB--the Tandem Repeats Database.

376 Nucleic Acids Res 35, D80-87 (2007).

377 26. Robinson, J.T. et al. Integrative genomics viewer. Nat Biotechnol 29, 24-26 (2011).

378

379

380 
a
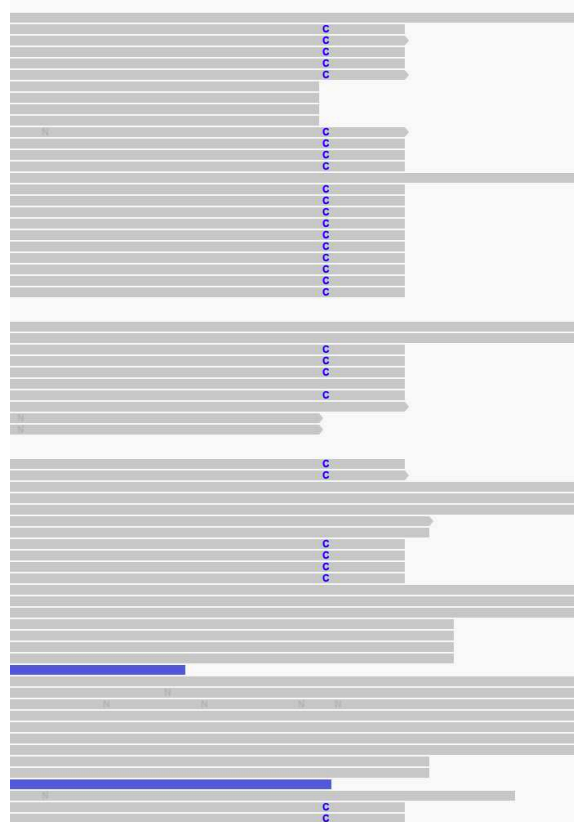

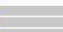
TOTGGOGTGCAGACATGAGCAAGGCTCAAGGCTGTOCAGGCACATA b

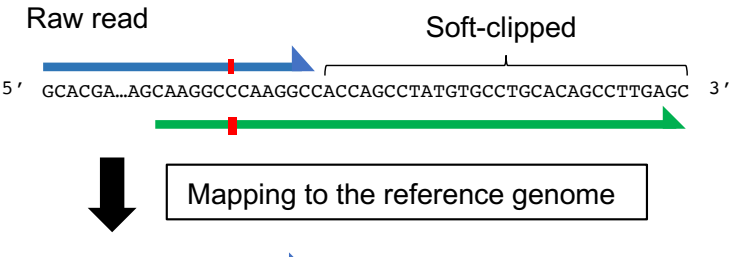

5. GCACGA...AGCAAGGCICAAGGC TGTGCAGGCACATAGGCTGGT GGCCI"IGGGCCI"TGG... 3

' CGTGCT....TCGTTCCGAGTTCCGACACGTCCGTGTATCCGACCAdCGGAACCCGGAACC...

C

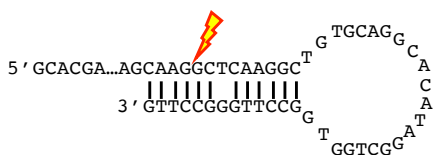

Nicking and partial denaturation

5' GCACGA AGCAAG 3'

3. GITCCGGGTTCCGGTGGTCGGATACACGGACGTGTCGGAACTCG 5'

\section{End repair}

5. GCACGA...AGCAAGGCCCAAGGCCACCAGCCTATGtgCCTGCACAGCCTTGAGC 3 '

3' CGTGCT...TCGTTCCGGGTTCCGGTGGTCGGATACACGGACGTGTCGGAACTCG 5'

d

Mutation position is too near to the effective end in supporting reads.

Palindromic sequences must exist in the same DNA fragment.

Mutations derived from distant

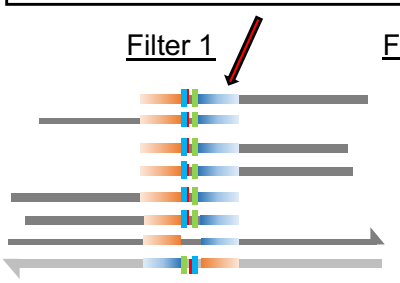

Filter 3

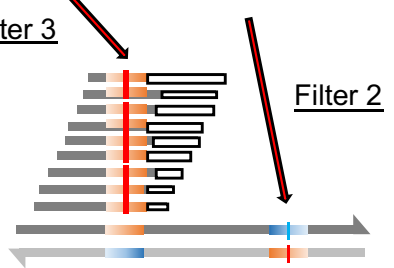

Homologous sequences

Mismatced bases

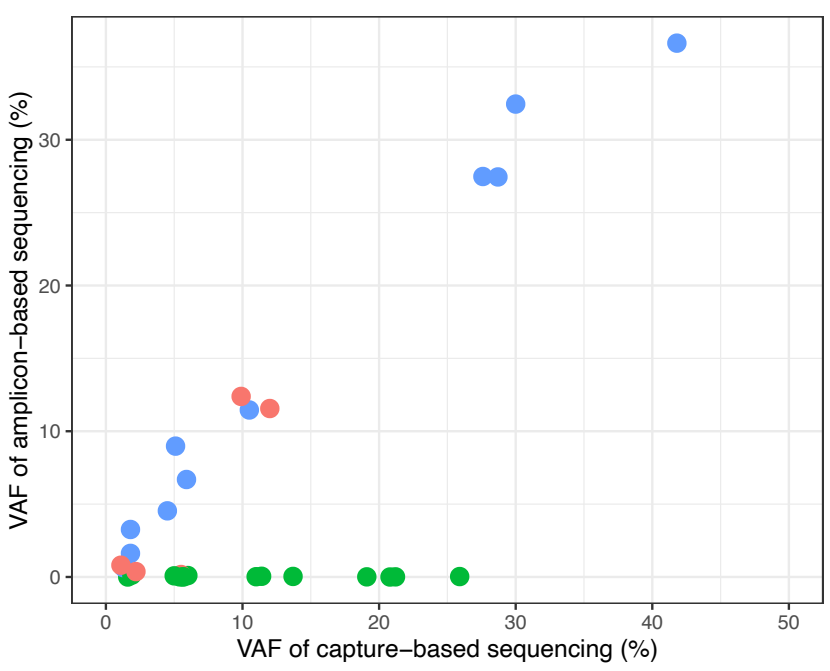
homologous regions.

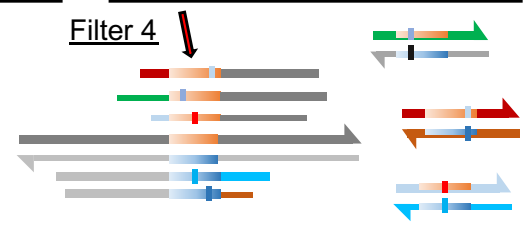

- Soft-clipped sequence
Filter passing mutation

CG-to-TG mutation

Filtered out mutation 
383 Figure 1. An example of microhomology-induced chimeric read (MICR)-originated sequencing error.

385 a. The genomic sequence visualized by Integrative Genomics Viewer exhibits a T-to-C

386 artifact in the FGFR4 gene. In all mutation-supporting reads, only six bases downstream of

387 the mutation were mapped, and the rest is soft-clipped.

388 b. A representative read supporting the mutation. The upstream sequence was mapped to the

389 forward strand (blue arrow), and the downstream sequence was mapped to the reverse strand

390 (green arrow). The red box represented the T-to-C artifact. Most of the downstream bases

391 were soft-clipped.

392 c. Two palindromic sequences in a single-stranded DNA (ssDNA) formed a hairpin structure.

393 After nicking and partial denaturation, the double-stranded DNA was regenerated during the

394 end-repair step of library preparation. The mismatched base between two palindromic

395 sequences was defined as a mutation.

396 d. The MicroSEC algorithm is based on three criteria. Filter 1, 3: the distance from the

397 mutation position to the most distant mapped base is distributed over a limited range for any

398 reads. Filter 2: MICR-originated sequencing errors are generated when two palindromic

399 sequences are in the same DNA fragment. Filter 4: The mis-annealing of ssDNA derived

400 from two distant homologous regions also creates an artifact.

401 e. Mutations identified by capture-based sequencing were validated by amplicon-based 
402 sequencing. The mutations that passed through the MicroSEC filter were detected with a

403 similar level of variant allele frequency (VAF) by both methods (blue). A potential CG-to-TG

404 artifact with a VAF of 5.5\% was not amplified by amplicon-based sequencing (red). Filtered-

405 out mutations were not detected by amplicon-based sequencing (green).

406 
Table Legend

\begin{tabular}{|c|c|c|c|c|c|}
\hline & \multicolumn{2}{|l|}{ Normal Breast Tissue } & \multicolumn{2}{|l|}{ Breast cancer } & \multirow{2}{*}{$\begin{array}{l}\text { Clinical sequencing } \\
\text { FFPE }(N=54)\end{array}$} \\
\hline & Fresh frozen $(\mathrm{N}=53)$ & FFPE $(N=190)$ & Fresh frozen $(\mathrm{N}=23)$ & FFPE $(N=33)$ & \\
\hline Total reads (in millions) & $61.3(36.6-120.4)$ & $83.5(37.3-154.8)$ & $60.8(43.3-114.2)$ & $67.5(20.0-127.6)$ & $87.8(38.0-216.0)$ \\
\hline Mapped reads (\%) & $92.2(89.7-93.7)$ & $93.4(89.4-96.8)$ & $91.9(88.7-93.6)$ & $92.3(89.0-94.3)$ & $90.5(85.9-92.9)$ \\
\hline Unique reads (\%) & $77.1(55.9-87.9)$ & $51.1(32.6-79.8)$ & $76.5(51.9-84.9)$ & $55.3(38.6-86.6)$ & $41.1(17.8-77.2)$ \\
\hline Mean coverage & $928(417-1,584)$ & $919(438-1,544)$ & $944(550-1,880)$ & $767(403-1,525)$ & 817 (404-1589) \\
\hline Median insert size (base) & $217(163-312)$ & $158(117-196)$ & 202 (158-293) & $142(119-173)$ & $135(104-177)$ \\
\hline $\begin{array}{l}\text { Somatic mutations } \\
\text { removed by }\end{array}$ & $0.3(0-9)$ & $11.8(0-117)$ & $4.0(0-13)$ & $10.8(2-34)$ & $21.7(1-135)$ \\
\hline Filter 1 & $0(0-0)$ & $4.3(0-88)$ & $0(0-0)$ & $1.0(0-7)$ & $0.5(0-12)$ \\
\hline Filter 2 & $0(0-0)$ & $2.3(0-29)$ & $0(0-0)$ & $0.7(0-4)$ & $0.1(0-1)$ \\
\hline Filter 3 & $0(0-0)$ & $3.6(0-76)$ & $0(0-0)$ & $0.8(0-6)$ & $0.7(0-17)$ \\
\hline Filter 4 & $0(0-0)$ & $5.4(0-40)$ & $0(0-0)$ & $1.8(0-9)$ & $3.2(0-89)$ \\
\hline Any of Filter 1-4 & $0(0-0)$ & $10.1(0-111)$ & $0(0-0)$ & $3.2(0-15)$ & $3.7(0-94)$ \\
\hline Mutations passing the filter & $0.3(0-9)$ & $1.7(0-50)$ & $4.0(0-13)$ & $7.5(0-32)$ & $18.0(0-135)$ \\
\hline Filtered rate (\%) & 0 & 85.8 & 0 & 30.1 & 16.9 \\
\hline CG-to-TG potential artifacts & NA & $0.1(0-12)$ & NA & $0.2(0-1)$ & $2.6(0-37)$ \\
\hline Intra $\geq 10$-base homopolymer & $0.0(0-1)$ & $0.7(0-14)$ & $0.0(0-0)$ & $0.2(0-2)$ & $0.1(0-1)$ \\
\hline Remaining mutations & $0.3(0-9)$ & $0.8(0-38)$ & $4.0(0-13)$ & $7.2(0-32)$ & $15.3(0-128)$ \\
\hline
\end{tabular}

Data are shown as mean (range).

NA, not applicable; FFPE, formalin-fixed and paraffin-embedded.

409 Table 1. MicroSEC filtering summary. 


\section{Supplementary Figure Legends}
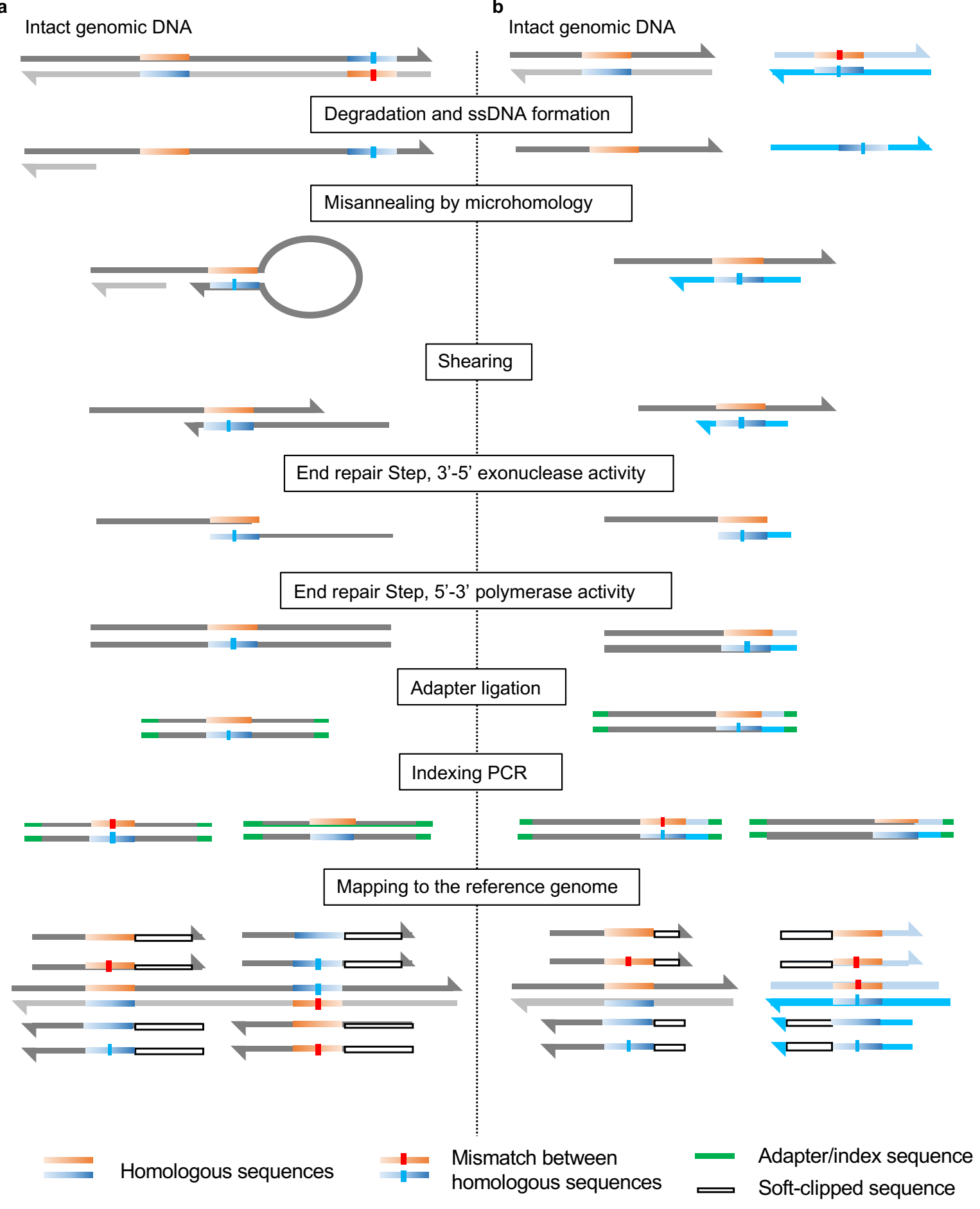

412

413 Supplementary Figure 1. Two proposed mechanisms of microhomology-induced chimeric

414 read (MICR) formation.

415 a. In the region with two proximal palindromic sequences, single-stranded DNA (ssDNA) is 
416 formed after degradation and denaturation. The annealing between the two palindromic

417 sequences forms a hairpin structure. The chimeric double-stranded DNA is formed during the

418 shearing and end-repair steps of library preparation. Adapter and index sequences are added to

419 the original sequence. The ligation product is amplified by polymerase chain reaction (PCR),

420 sequenced, and mapped to the reference genome. In a chimeric read of two sequences, only

421 one is correctly mapped, and the other is clipped by the mapping software.

422 b. MICR is also formed by the homologous sequences in two distant regions in the genome.

423 Chimeric reads are generated by mis-annealing of ssDNA derived from the distant homologous

424 regions.

425 
a
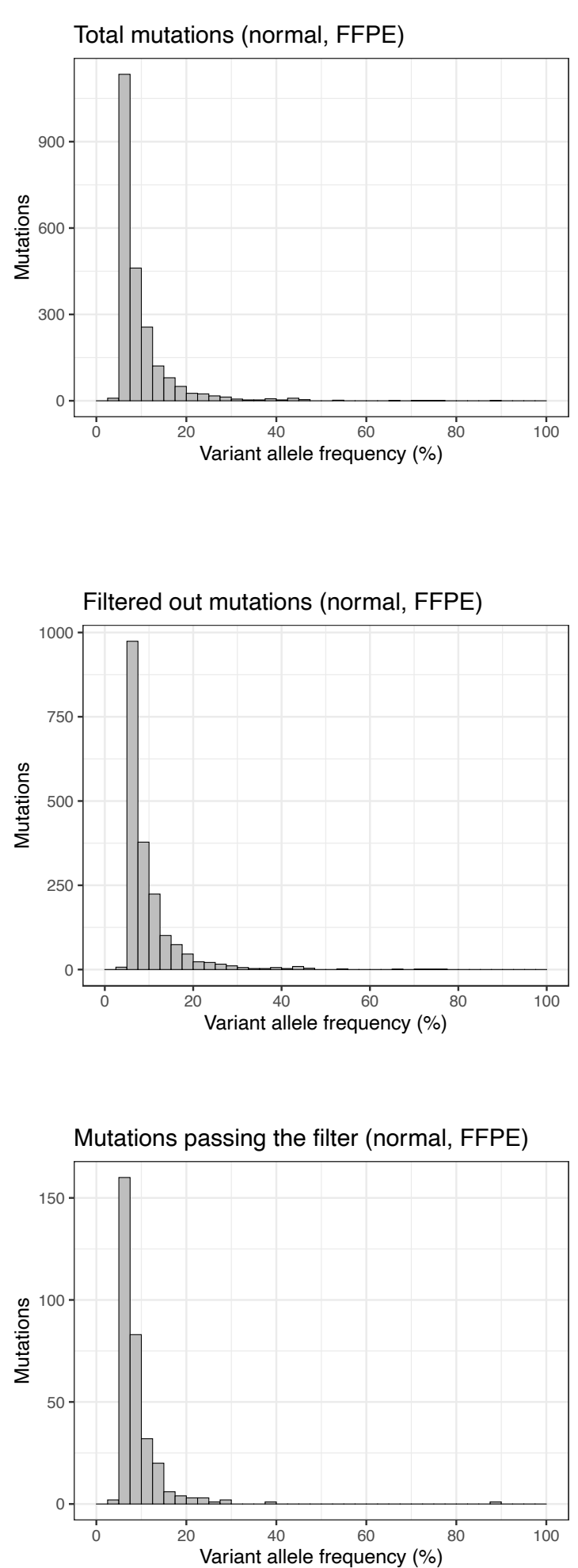

b

Total mutations (tumor, FFPE)

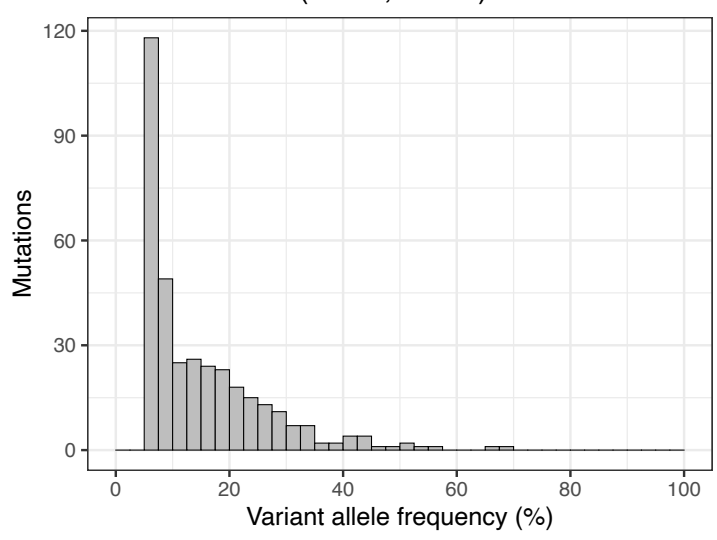

Filtered out mutations (tumor, FFPE)

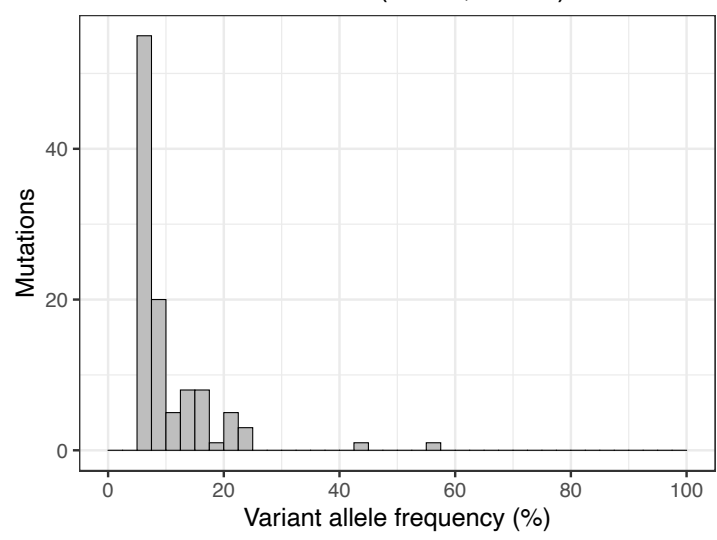

Mutations passing the filter (tumor, FFPE)

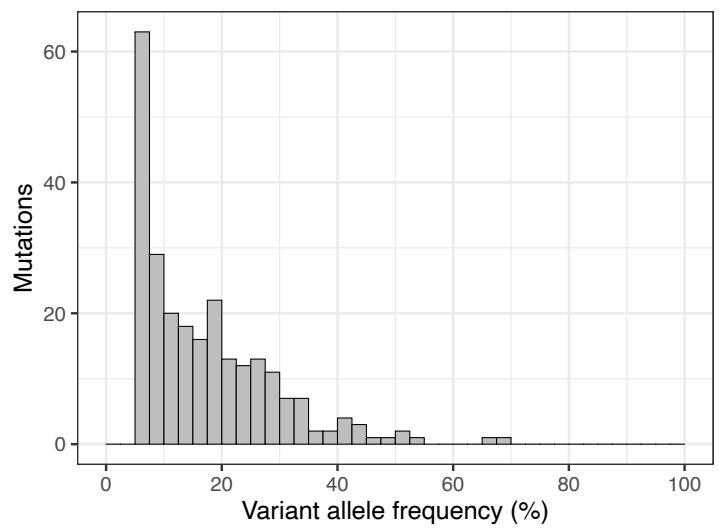


430 a. FFPE samples of normal breast tissues $(\mathrm{n}=190)$ with total mutations (upper), mutations

431 filtered out by MicroSEC filter (middle), and mutations passing through the filter (lower).

432 b. FFPE samples of breast tumor tissues $(n=33)$ with total mutations (upper), mutations

433 filtered out by MicroSEC filter (middle), and mutations passed through the filter (lower).

434 FFPE, formalin-fixed and paraffin-embedded.

435 


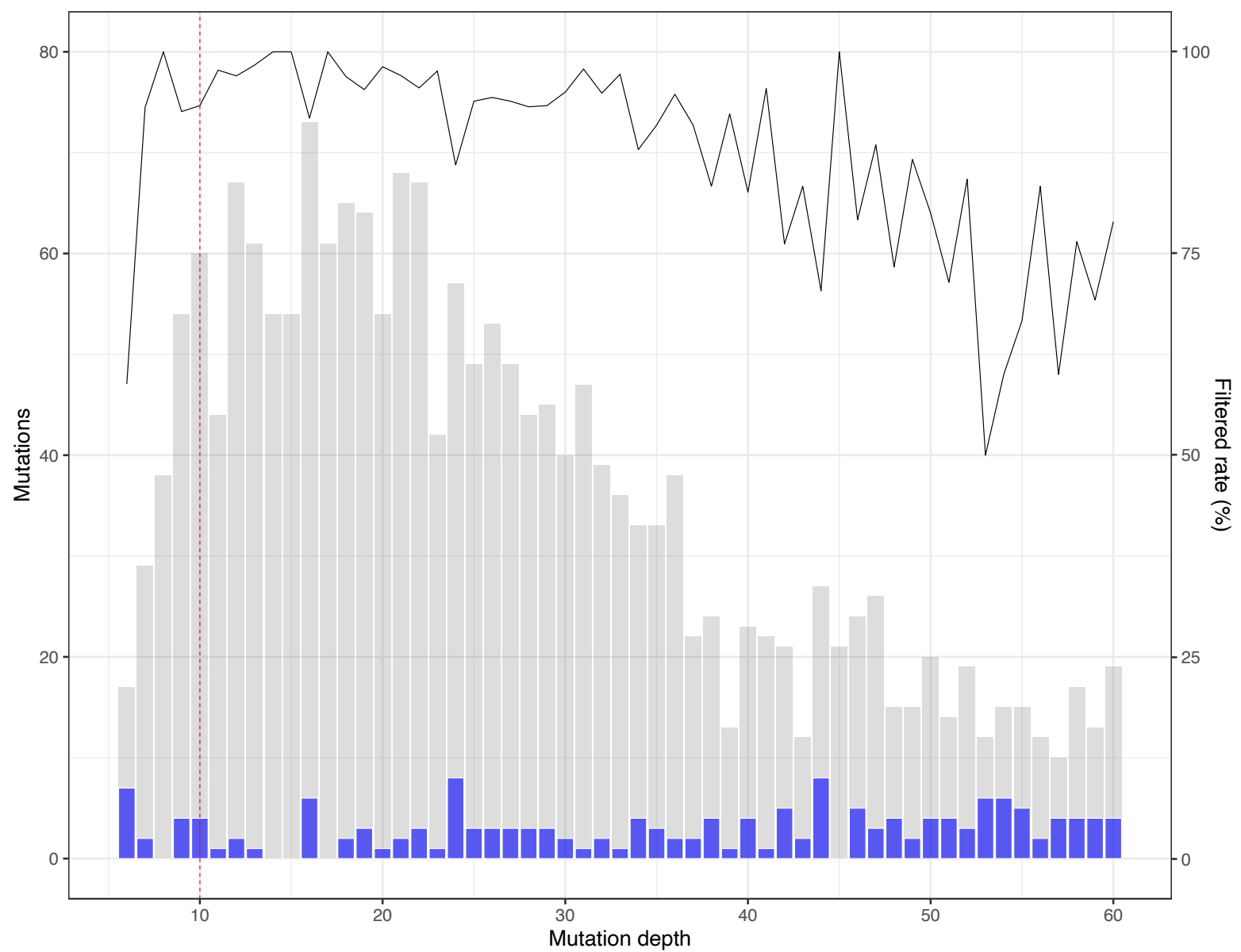

436

437 Supplementary Figure 3. The relationship between the mutation depth and filtering

438 efficiency in the 190 FFPE normal breast tissue samples.

439 The histograms of mutation depth for total mutations (gray) and mutation passing the

440 MicroSEC filter (blue) were shown. Most of the called mutations are considered to be artifacts,

441 because normal breast tissues have little or no mutations. The mutation filtering rate (black

442 line) was not high at low mutation depth, and more than $90 \%$ of mutations were filtered by the

443 MicroSEC pipeline when the mutation coverage was 10 or larger (dotted red line). FFPE,

444 formalin-fixed and paraffin-embedded. 
Matching samples of breast cancer

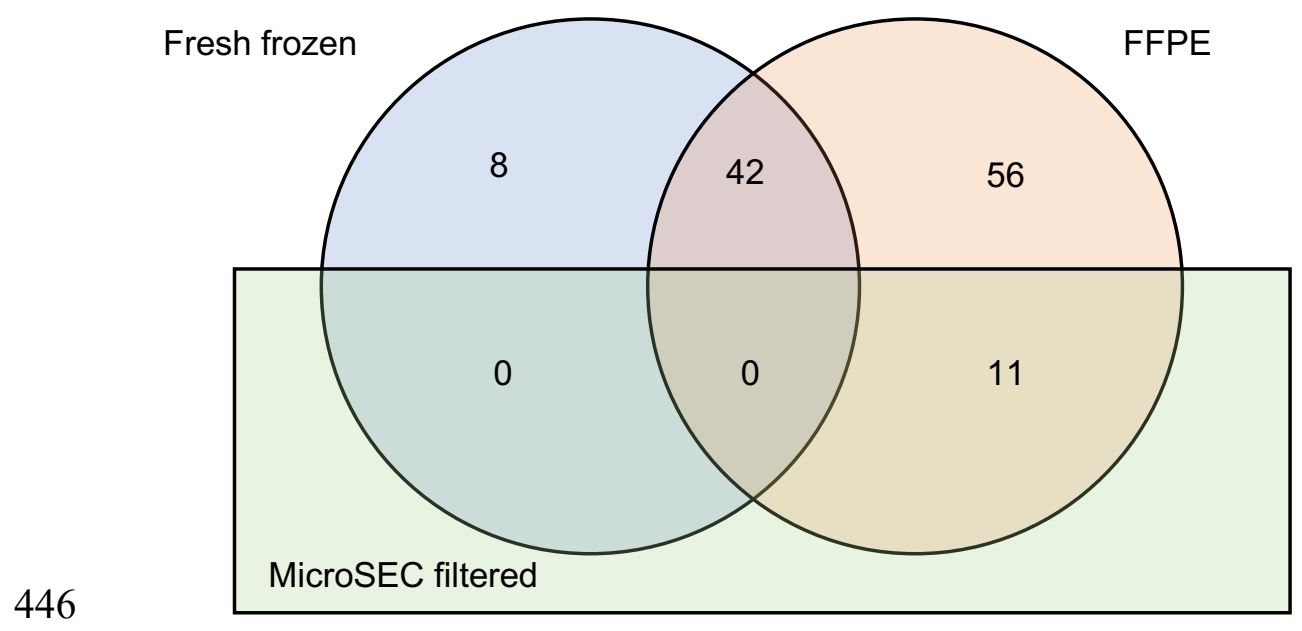

447 Supplementary Figure 4. The mutations detected in matching FF and FFPE samples.

448 The mutations detected in the matching sets of frozen (blue) and FFPE breast cancer (peach)

449 samples from eight patients. Eleven mutations found in only FFPE samples were filtered by

450 MicroSEC (green). All mutations found in fresh frozen samples passed through the MicroSEC

451 filter. FFPE, formalin-fixed and paraffin-embedded.

452 


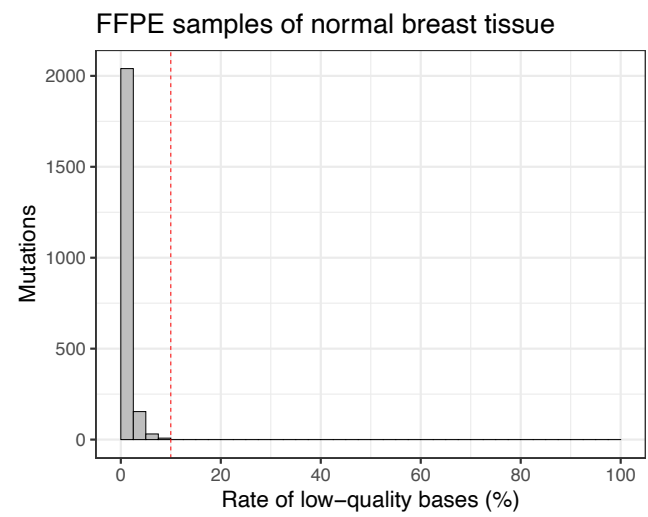

b

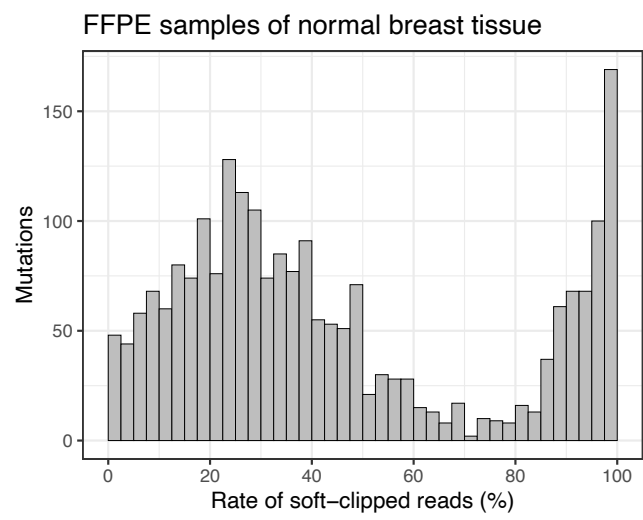

C

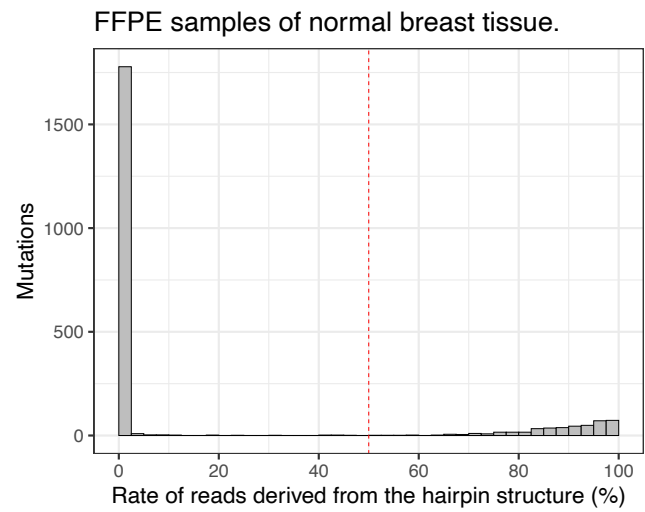

d
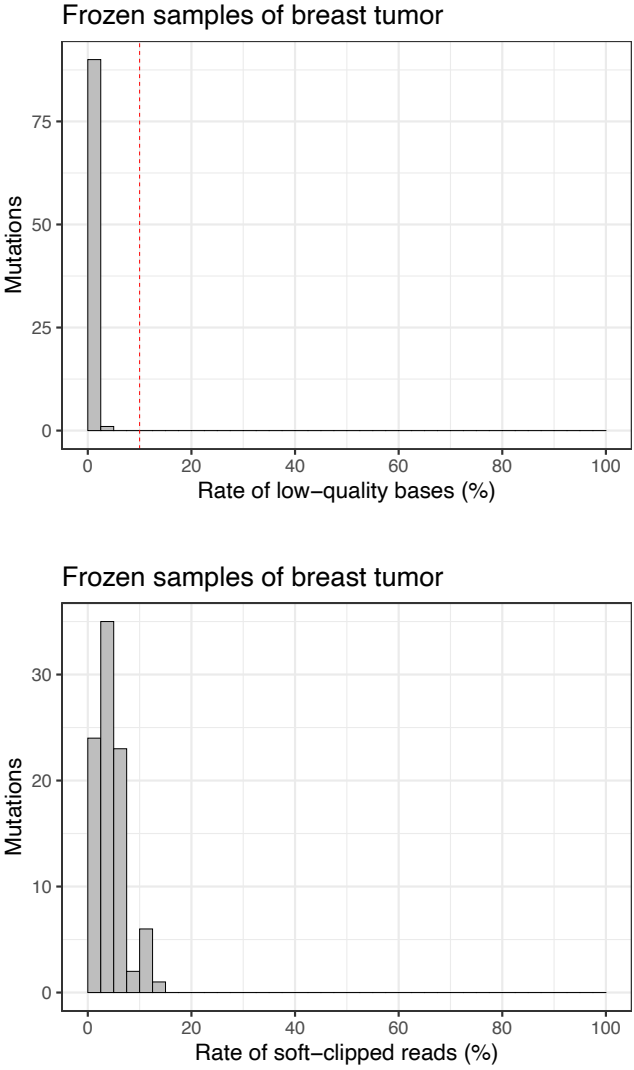

Frozen samples of breast tumor.

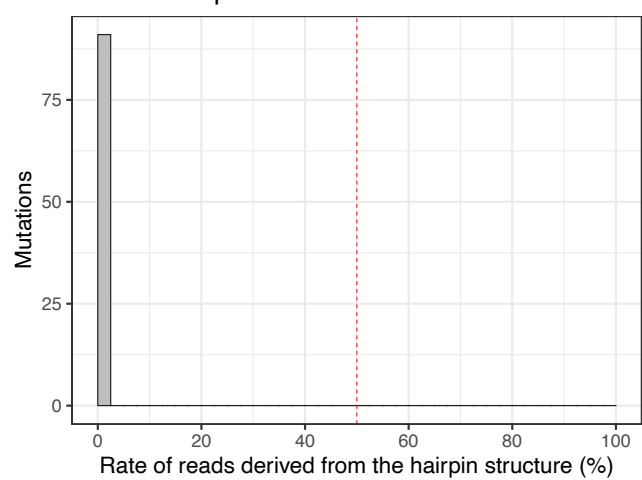

Frozen samples of breast tumor

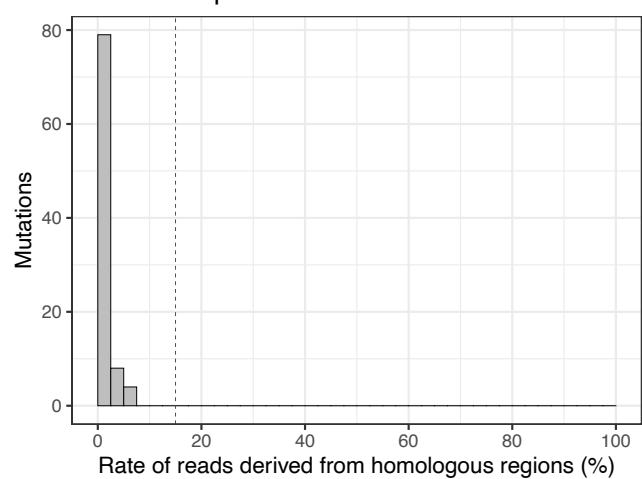

454 Supplementary Figure 5. The distribution of the mutations in breast tissue samples. 
455 a. The rate of low-quality bases in mutation-supporting reads in 190 FFPE normal breast tissue

456 samples (left) and 23 frozen breast tumor samples (right).

457 b. The rate of soft-clipped reads in FFPE samples of normal breast tissue (left) and frozen 458 samples of breast tumor (right).

459 c. The rate of reads derived from other homologous regions in FFPE samples of normal breast 460 tissue (left) and frozen samples of breast tumor (right).

$461 \mathrm{~d}$. The rate of reads derived from the hairpin structure in FFPE samples of normal breast tissue

462 (left) and frozen samples of breast tumor (right).

463 Dotted red lines represent the thresholds.

464 
465 Supplementary Table Legends

\begin{tabular}{|c|c|c|c|c|c|c|c|c|c|c|c|c|c|c|c|}
\hline \multirow{2}{*}{ Sample } & \multirow{2}{*}{ Chr } & \multirow{2}{*}{ Position } & \multirow{2}{*}{ Ref } & \multirow{2}{*}{ Alt } & \multirow{2}{*}{$\begin{array}{c}\text { VAF } \\
(\%)\end{array}$} & \multirow{2}{*}{$\begin{array}{r}\text { Mutation } \\
\text { depth }\end{array}$} & \multirow{2}{*}{$\begin{array}{r}\text { Soft-clipped } \\
\text { read }\end{array}$} & \multirow{2}{*}{$\begin{array}{l}\text { Hairpin- } \\
\text { structure }\end{array}$} & \multicolumn{4}{|c|}{ Read Supporting length } & \multicolumn{3}{|c|}{ Probability } \\
\hline & & & & & & & & & length ${ }^{-}$ & 5 , & $3^{\prime}$ & Shorter & 5 & $3{ }^{\prime}$ & Shorter \\
\hline SL_0001_L_FFPE_4 & 12 & 106255381 & $G$ & GTGAC & 55.4 & 652 & $645(98.9 \%)$ & $632(96.9 \%)$ & 150 & 145 & 125 & 10 & NA & NA & 0 \\
\hline SL_1003_R_FFPE_66 & 12 & 106255382 & A & $\mathrm{T}$ & 70.2 & 536 & $474(88.4 \%)$ & $523(97.6 \%)$ & 150 & 148 & 136 & 36 & NA & NA & $3.0 \times 10^{-25}$ \\
\hline SL_1008_L_FFPE_13 & 12 & 106255382 & A & T & 74.3 & 916 & $807(88.1 \%)$ & 899 (98.1\%) & 150 & 149 & 136 & 37 & NA & NA & $1.4 \times 10^{-34}$ \\
\hline SL_1008_R_FFPE_32 & 12 & 106255382 & A & T & 53.4 & 378 & $335(88.6 \%)$ & 368 (97.3\%) & 150 & 147 & 139 & 38 & NA & NA & $1.4 \times 10^{-24}$ \\
\hline SL_1008_R_FFPE_50 & 12 & 106255382 & A & T & 75.9 & 950 & $880(92.6 \%)$ & 935 (98.4\%) & 150 & 149 & 136 & 37 & NA & NA & $3.9 \times 10^{-34}$ \\
\hline SL_1015_R_69_FFPE & 12 & 106255382 & A & T & 65.4 & 461 & 407 (88.2\%) & 447 (97.0\%) & 124 & 123 & 110 & 59 & NA & NA & NA \\
\hline
\end{tabular}

Chr, chromosome; Ref, reference sequence; Alt, altered sequence; VAF, variant allele frequency; NA, not assessed.

Probability is calculated only if the supporting length is $<80 \%$ of the read length.

466

467 Supplementary Table 1. Filtered mutations with high variant allele frequency in FFPE

468

samples.

469 


\begin{tabular}{|c|c|c|c|c|c|c|c|c|c|c|c|}
\hline \multirow{3}{*}{$\begin{array}{l}\text { Sample } \\
\text { SL_1006_L_FFPE_16 }\end{array}$} & \multirow{3}{*}{$\begin{array}{r}\text { Chr } \\
1\end{array}$} & \multirow{3}{*}{$\frac{\text { Pos }}{36469724 \mathrm{G}}$} & \multirow[b]{2}{*}{ Ref Alt } & \multirow[b]{2}{*}{ Gene } & \multirow[b]{2}{*}{ HGVS.p } & \multicolumn{3}{|c|}{ Capture-based sequencing } & \multicolumn{3}{|c|}{ Amplicon-based sequencing } \\
\hline & & & & & & \multirow{2}{*}{\multicolumn{2}{|c|}{\begin{tabular}{|rr}
\multicolumn{2}{|c|}{ Total } \\
read & depth \\
668 & 36 deption \\
\end{tabular}}} & \multirow{2}{*}{$\begin{array}{l}\begin{array}{l}\text { VAF } \\
(\%)\end{array} \\
5.4 \text { Filter } 1,3\end{array}$} & \multirow{2}{*}{$\begin{array}{r}\text { Total read } \\
21,677\end{array}$} & \multicolumn{2}{|c|}{$\begin{array}{c}\text { Mutation VAF (\%) } \\
\text { depth }\end{array}$} \\
\hline & & & T & CSF3R & p.Pro468Thr & & & & & 3 & 0.01 \\
\hline SL_1006_L_FFPE_22 & 1 & $36469724 \mathrm{G}$ & T & CSF3R & p.Pro468Thr & 555 & 61 & 11 Filter 1, 3 & 33,998 & 6 & 0.02 \\
\hline SL_0001_R_FFPE_58 & 1 & $43360798 \mathrm{~T}$ & A & CDC20 & p.Leu305GIn & 104 & 22 & 21.2 Filter 1-3 & 37,241 & 4 & 0.01 \\
\hline SL_0006_RN_4 & 3 & 52403601 TT & GG & BAP1 & p.Asn515Pro & 1,825 & 29 & 1.6 Filter 3 & 156,752 & 0 & 0.00 \\
\hline SL_0007_R_FFPE_48 & 3 & $179199690 \mathrm{G}$ & A & PIK3CA & p.Gly118Asp & 1,046 & 19 & 1.8 Passed & 55,234 & 709 & 1.62 \\
\hline SL_1010-N4 & 3 & $179218294 \mathrm{G}$ & A & PIK3CA & p.Glu542Lys & 599 & 172 & 28.7 Passed & 132,576 & 36,391 & 27.45 \\
\hline SL_1010-N5 & 3 & $179218294 \mathrm{G}$ & A & PIK3CA & p.Glu542Lys & 780 & 215 & 27.6 Passed & 155,872 & 42,841 & 27.48 \\
\hline SL_0007_LT_9 & 3 & $179234297 \mathrm{~A}$ & G & PIK3CA & p.His1047Arg & 897 & 12 & 1.3 Passed & 48,955 & 312 & 0.64 \\
\hline SL_0008_LT_8 & 3 & 179234297 A & G & PIK3CA & p.His1047Arg & 958 & 101 & 10.5 Passed & 47,909 & 5,492 & 11.46 \\
\hline SL_1007_L_FFPE_26 & 3 & $179234297 \mathrm{~A}$ & G & PIK3CA & p.His1047Arg & 901 & 46 & 5.1 Passed & 49,568 & 4,452 & 8.98 \\
\hline SL_1004_R_49_FFPE & 3 & 179234297 A & G & PIK3CA & p.His1047Arg & 641 & 268 & 41.8 Passed & 115,256 & 42,213 & 36.63 \\
\hline SL0001_L_FFPE_10 & 3 & 179234297 A & G & PIK3CA & p.His1047Arg & 554 & 10 & 1.8 Passed & 50,261 & 1,638 & 3.26 \\
\hline SL0001_R_FFPE_58 & 3 & $179234297 \mathrm{~A}$ & G & PIK3CA & p.His1047Arg & 491 & 22 & 4.5 Passed & 48,777 & 2,216 & 4.54 \\
\hline SL_0001_L_FFPE_3 & 6 & $137881182 \mathrm{G}$ & A & TNFAIP3 & p.Gly746Arg & 553 & 63 & 11.4 Filter 1,2 & 16,044 & 8 & 0.05 \\
\hline SL_1007_T2 & 6 & $137881182 \mathrm{G}$ & A & TNFAIP3 & p.Gly746Arg & 384 & 23 & 6 Filter 2 & 8,099 & 8 & 0.10 \\
\hline SL_1010-N2 & 6 & $152011697 \mathrm{G}$ & C & ESR1 & p.Glu380Gln & 1,332 & 182 & 13.7 Filter 3 & 83,395 & 32 & 0.04 \\
\hline SL_1004_R_FFPE_61 & 6 & $152011739 \mathrm{C}$ & T & ESR1 & p.Arg394Cys & 785 & 43 & 5.5 Passed $(\mathrm{Cg}>\mathrm{Tg})$ & 146,849 & 248 & 0.17 \\
\hline SL_0001_L_FFPE_10 & 12 & $68815634 \mathrm{~A}$ & G & MDM2 & p.Ser55Gly & 189 & 49 & 25.9 Filter 4 & 3,604 & 1 & 0.03 \\
\hline SL_0012_RT_5 & 12 & $12745786 \mathrm{~A}$ & $\mathrm{G}$ & APOLD1 & - & 1,065 & 20 & 1.9 Filter 1 & 138,907 & 179 & 0.13 \\
\hline SL_1010_L_FFPE_6 & 13 & $32356550 \mathrm{C}$ & T & BRCA2 & p.Arg2520* & 540 & 12 & 2.2 Passed $(\mathrm{Cg}>\mathrm{Tg})$ & 36,182 & 134 & 0.37 \\
\hline SL_1010_L_FFPE_9 & 13 & $32356550 \mathrm{C}$ & $\mathrm{T}$ & BRCA2 & p.Arg2520* & 651 & 7 & 1.1 Passed $(\mathrm{Cg}>\mathrm{Tg})$ & 40,544 & 328 & 0.81 \\
\hline SL_1007-T2 & 13 & 48360023 TATTAC & $\mathrm{C} T$ & RB1 & p.Leu206fs & 347 & 104 & 30 Passed & 213,320 & 69,215 & 32.45 \\
\hline SL_0001_R_FFPE_52 & 14 & $36519170 \mathrm{G}$ & A & NKX2-1 & p.Ala63Val & 741 & 42 & 5.7 Filter 4 & 42,628 & 3 & 0.01 \\
\hline SL_0001_L_FFPE_29 & 14 & 68594559 C & T & RAD51B & p.GIn371* & 538 & 101 & 19.1 Filter 4 & 74,485 & 7 & 0.01 \\
\hline SL_1010-T1 & 17 & $7670685 \mathrm{G}$ & $A$ & TP53 & p.Arg342* & 707 & 85 & 12 Passed $(\mathrm{Cg}>\mathrm{Tg})$ & 85,333 & 9,867 & 11.56 \\
\hline SL_1004_L_36_FFPE & 17 & $7673803 \mathrm{G}$ & $A$ & TP53 & p.Arg273Cys & 729 & 72 & 9.9 Passed $(\mathrm{Cg}>\mathrm{Tg})$ & 197,963 & 24,528 & 12.39 \\
\hline SL_0001_R_FFPE_56 & 17 & $7674385 \mathrm{~A}$ & $\mathrm{G}$ & TP53 & - & 1466 & 82 & 5.6 Filter 4 & 8,742 & 1 & 0.01 \\
\hline SL_1006_L_FFPE_22 & 19 & $6828091 \mathrm{G}$ & A & VAV1 & p.Ala315Thr & 298 & 15 & 5 Filter 1,3 & 17,710 & 14 & 0.08 \\
\hline SL_0007_L_FFPE_19 & 19 & $46919618 \mathrm{C}$ & T & ARHGAP35 & p.GIn315* & 1,130 & 67 & 5.9 Passed & 28,581 & 1,911 & 6.69 \\
\hline SL_1007-T2 & $x$ & $15801329 \mathrm{CA}$ & $A G$ & ZRSR2 & p.His87Ser & 197 & 41 & 20.8 Filter 4 & 2,329 & 0 & 0.00 \\
\hline
\end{tabular}

470 Chr, chromosome; Ref, reference sequence; Alt, altered sequence; HGVS, Human Genome Variation Society; VAF, variant allele frequency.

471 Supplementary Table 2. Comparison between the results of capture-based sequencing

472 and amplicon-based sequencing.

473 


\begin{tabular}{|c|c|c|c|c|c|c|c|c|c|c|c|c|}
\hline \multirow{2}{*}{ Sample } & \multirow{2}{*}{ Gene } & \multirow{2}{*}{ HGVS.C } & \multirow{2}{*}{ HGVS.p } & \multirow{2}{*}{$\begin{array}{r}\text { VAF } \\
(\%)\end{array}$} & \multirow{2}{*}{$\begin{array}{r}\text { Mutation } \\
\text { depth }\end{array}$} & \multirow{2}{*}{$\begin{array}{r}\text { Soft-clipped } \\
\text { read }\end{array}$} & \multicolumn{4}{|c|}{ Read Supporting length } & \multirow{2}{*}{$\begin{array}{r}\text { Reads from } \\
\text { distant region }\end{array}$} & \multirow{2}{*}{ MicroSEC } \\
\hline & & & & & & & length & $5^{\prime}$ & $3^{\prime}$ & Shorter & & \\
\hline H12-13366-21 & RAD51B & c. $1111 \mathrm{C}>\mathrm{T}$ & p.GIn $371^{*}$ & 12.2 & 24 & $13(54.2 \%)$ & 150 & 143 & 39 & 39 & $10(41.7 \%)$ & Filter 4 \\
\hline H17-09165-20 & PPP2R1A & c.108delT & p.Leu36fs & 13.4 & 17 & $5(29.4 \%)$ & 150 & 46 & 141 & 46 & $9(52.9 \%)$ & Filter 4 \\
\hline H17-06211-11 & FAM175A & c. $229 \mathrm{C}>\mathrm{T}$ & p.Arg77* & 11.8 & 16 & $1(6.3 \%)$ & 151 & 141 & 68 & 68 & $11(68.8 \%)$ & Filter 4 \\
\hline H17-06211-11 & RAD51B & c. $1111 \mathrm{C}>\mathrm{T}$ & p.G $\ln 371^{*}$ & 10.9 & 57 & $24(42.1 \%)$ & 151 & 150 & 50 & 50 & $22(38.6 \%)$ & Filter 4 \\
\hline H17-06211-11 & PPP2R1A & c.108delT & p.Leu36fs & 21.1 & 23 & $6(26.1 \%)$ & 151 & 63 & 145 & 63 & $15(65.2 \%)$ & Filter 4 \\
\hline H17-09880-1 & TP53 & c. $1021 T>G$ & p.Phe341Val & 8.8 & 62 & $4(6.5 \%)$ & 151 & 102 & 150 & 75 & $0(0 \%)$ & Filter 3 \\
\hline H17-10196-1 & PPP2R1A & c.108delT & p.Leu $36 \mathrm{fs}$ & 9.3 & 23 & $6(26.1 \%)$ & 151 & 52 & 144 & 52 & $13(56.5 \%)$ & Filter 4 \\
\hline H17-10196-1 & ZRSR2 & c. $283 C>T$ & p.Arg95* & 6.0 & 10 & $5(50 \%)$ & 151 & 149 & 115 & 55 & $6(60 \%)$ & Filter 4 \\
\hline
\end{tabular}

Chr, chromosome; Ref, reference sequence; Alt, altered sequence; NA, not assessed.

Probability is calculated only if the supporting length is $<80 \%$ of the read length.

474

475 Supplementary Table 3. Pathogenic mutations in clinical FFPE samples filtered out by

476 MicroESC.

477 


\begin{tabular}{|c|c|c|c|c|c|c|c|c|}
\hline \multicolumn{6}{|c|}{ Target region } & \multirow{2}{*}{$\begin{array}{l}\text { Insert } \\
\text { bases) }\end{array}$} & \multirow{2}{*}{ Forward primer } & \multirow{2}{*}{ Reverse primer } \\
\hline Chr & Pos & Ref & Alt & Gene & $\overline{\text { HGVS.p }}$ & & & \\
\hline 1 & 36469724 & $G$ & $\mathrm{~T}$ & CSF3R & p.Pro468Thr & 114 & TTCAGCAGAAACCCCGTGGC & $\overline{\text { TCCATGGCCTCAGGGCTATG }}$ \\
\hline 1 & 43360798 & $\mathrm{~T}$ & A & $\mathrm{CDC} 20$ & p.Leu305GIn & 128 & TGGTTCACGTTCTGGCCACATC & GACCAAGTTATCATTACCACCACTG \\
\hline 3 & 52403601 & $\mathrm{TT}$ & GG & BAP1 & p.Asn515Pro & 136 & GCTGTCATCCTCTCCAAAAAGCAC & TACAGACACGGCCTCTGAGATC \\
\hline 3 & 179199690 & G & A & PIK3CA & p.Gly118Asp & 137 & GTTTGCAAAAAAAACATGTTCATGCTGTG & GGAAGTCCTGTACTTCTGGATC \\
\hline 3 & 179218294 & G & A & PIK3CA & p.Glu542Lys & 127 & CTAGAGACAATGAATTAAGGGAAAATGAC & CATTTTAGCACTTACCTGTGACTCC \\
\hline 3 & 179234297 & A & G & PIK3CA & p.His1047Arg & 136 & ACATTCGAAAGACCCTAGCCTTAG & ATGCTGTTTAATTGTGTGGAAGATCC \\
\hline 6 & 137881182 & G & A & TNFAIP3 & p.Gly746Arg & 156 & CAAGAACATCCTGGCCTGCC & GCATTGCCAAAATGATCACAGGC \\
\hline 6 & 152011697 & G & C & ESR1 & p.Glu380Gln & 119 & TGTTTTCAGGCTTTGTGGATTTGAC & TAGCTTCCCTGGGTGCTCCATG \\
\hline 6 & 152011739 & C & $\mathrm{T}$ & ESR1 & p.Arg394Cys & 122 & CTCCATGATCAGGTCCACCTTC & CTGTCCAAGAGCAAGTTAGGAG \\
\hline 12 & 12745786 & $A$ & G & APOLD1 & - & 106 & AGGATGACTCAGAATAGCACAAATAG & GAAAGATCTGCTGTAGGCAAGACC \\
\hline 12 & 68815634 & A & G & MDM2 & p.Ser55Gly & 149 & AGTGTCTGCAGTAGCTAGGACTAC & ACTTTAGGAGGCCGAGGTAAGCAG \\
\hline 13 & 32356550 & C & $\mathrm{T}$ & BRCA2 & p.Arg2520* & 153 & GAAGAAACAAAGGCAACGCGTC & CAGTATTGTAGACAAACACATACCTG \\
\hline 13 & 48360023 & TATTAC & $\mathrm{T}$ & RB1 & p.Leu206fs & 127 & TTTCTCTCATACAAAGATCTGAATCTC & AGTCAAGGACACATAGCATTAACTG \\
\hline 14 & 36519170 & G & A & NKX2-1 & p.Ala63Val & 125 & CAGGTTGCCGTTGCAGTAGC & CAGCGGCCATGCAGCAGCAC \\
\hline 14 & 68594559 & $\mathrm{C}$ & $\mathrm{T}$ & RAD51B & p.GIn $371^{*}$ & 126 & CCAAGCTGAACTGAACTGGGCTC & ACATAATGAGACCCCACCTCTAC \\
\hline 17 & 7670685 & G & A & TP53 & p.Arg342* & 148 & CTGAGGTCACTCACCTGGAGTGAG & АСТTCTСССССтССтСTGTTGCTG \\
\hline 17 & 7673803 & G & A & TP53 & p.Arg273Cys & 113 & GGCTCCCCTTTCTTGCGGAGATTC & ATCCTGAGTAGTGGTAATCTACTG \\
\hline 17 & 7674385 & A & G & TP53 & - & 124 & GGGGAGACCTGTGGCAAGCAG & ACCTGGGCGGTGGAGCTTGC \\
\hline 19 & 6828091 & G & A & VAV1 & p.Ala315Thr & 151 & CAACTGGCTGTTTCTGGGACC & TGGAGAAGGAGGTGATATTTGAGAAC \\
\hline 19 & 46919618 & C & $\mathrm{T}$ & ARHGAP35 & p.G $\ln 315^{*}$ & 148 & GGCTGAGTGTCAGCCGAAAG & GGTACAGCTTTCTCCTACGCTC \\
\hline$x$ & 15801329 & $\mathrm{CA}$ & $A G$ & ZRSR2 & p.His87Ser & 147 & TGTTTTGTTTGAGACGGACTCTCG & TGGCGGTGCGTGCCTGTAATC \\
\hline
\end{tabular}

Chr, chromosome; Ref, reference sequence; Alt, altered sequence; HGVS, Human Genome Variation Society.

\section{Supplementary Table 4. PCR primer sets used in this study.}




\section{Figures}

a

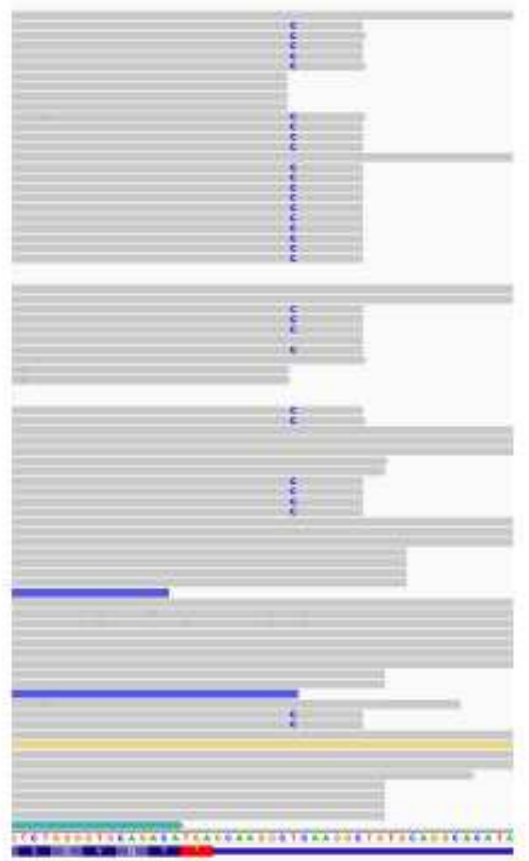

b

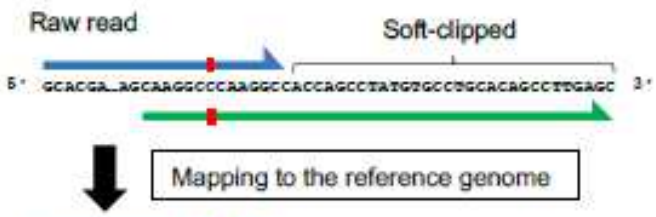

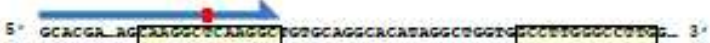

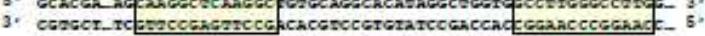

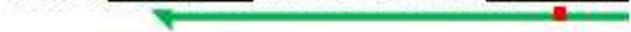

c

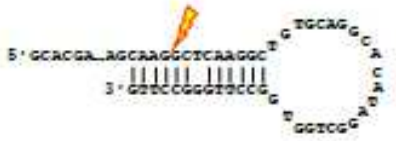

Nicking and partial denaturation

5. ecacca agcanc 3.

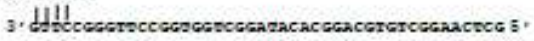

\section{End repair}

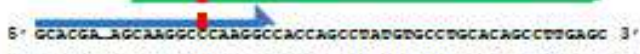

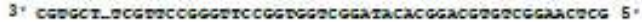

d

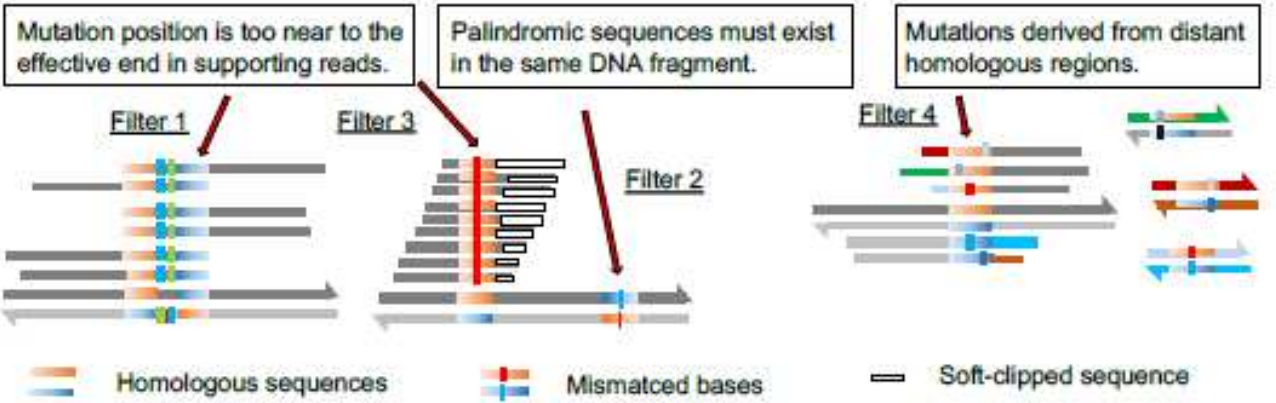

e

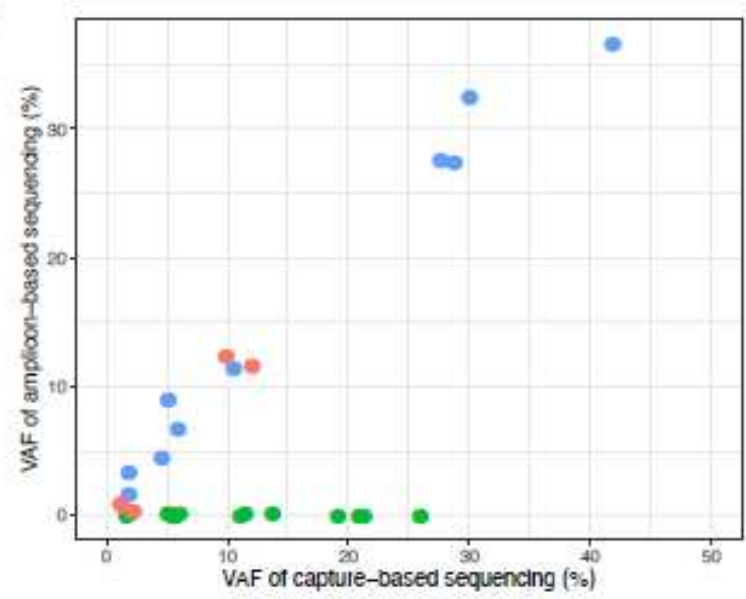

- Filter passing mutation

- CG-to-TG mutation

- Filtered out mutation

\section{Figure 1}

An example of microhomology-induced chimeric 383 read (MICR)-originated sequencing error. a. The genomic sequence visualized by Integrative Genomics Viewer exhibits a T-to-C artifact in the FGFR4 gene. In all mutation-supporting reads, only six bases downstream of the mutation were mapped, and the rest is 
soft-clipped. b. A representative read supporting the mutation. The upstream sequence was mapped to the forward strand (blue arrow), and the downstream sequence was mapped to the reverse strand (green arrow). The red box represented the T-to-C artifact. Most of the downstream bases were soft-clipped. $\mathrm{C}$. Two palindromic sequences in a single-stranded DNA (ssDNA) formed a hairpin structure. After nicking and partial denaturation, the double-stranded DNA was regenerated during the end-repair step of library preparation. The mismatched base between two palindromic sequences was defined as a mutation. $d$. The MicroSEC algorithm is based on three criteria. Filter 1, 3: the distance from the mutation position to the most distant mapped base is distributed over a limited range for any reads. Filter 2: MICR-originated sequencing errors are generated when two palindromic sequences are in the same DNA fragment. Filter 4: The mis-annealing of ssDNA derived from two distant homologous regions also creates an artifact. e. Mutations identified by capture-based sequencing were validated by amplicon-based sequencing. The mutations that passed through the MicroSEC filter were detected with a similar level of variant allele frequency (VAF) by both methods (blue). A potential CG-to-TG artifact with a VAF of $5.5 \%$ was not amplified by amplicon-based sequencing (red). Filtered out mutations were not detected by ampliconbased sequencing (green).

\section{Supplementary Files}

This is a list of supplementary files associated with this preprint. Click to download.

- supplementarydatalkegami.xlsx 